\title{
Analysis of the integration of indoor ecological landscape design and interior decoration design
}

\section{Qiu Zhenfeng}

Jiangxi Industrial Engineering Vocational and Technical College Pingxiang Jiangxi Province 337000

\author{
Keywords: Ecological Landscape Design; Decoration Design; Fusion
}

\begin{abstract}
With the improvement of the quality of life, people have gradually increased their requirements for interior design. In order to rationally use the interior space and save the interior decoration funds, the interior decoration design must rationally apply natural elements. Appropriate application of ecological landscape is the only means to enhance the effect of interior decoration and achieve environmental protection and conservation.
\end{abstract}

\section{Introduction}

With the development of modern design, people pay attention to the natural ecological aesthetic value. The integration of ecological landscape design into interior design fully demonstrates the aesthetic significance of interior design. It is the mainstream of the future development of interior decoration design, thus improves the quality of life of people. In this regard, the integration of indoor ecological landscape design and interior decoration design should be fully analyzed to improve the artistic and aesthetic sense of the indoor environment.

\section{Interior ecological landscape design and interior decoration design concept}

\subsection{Indoor ecological landscape design}

Creating a comfortable indoor environment is the focus of indoor ecological landscape design. To achieve this goal, we must integrate the indoor environment with the natural environment, so that the residents feel the wonders of the natural ecological environment and enjoy the comfort brought by nature.[2] At present, the study of indoor ecological landscape design has achieved initial success. There are obvious differences in people's perceptions. The following are some of the concepts of indoor ecological landscape design: (1) Indoor ecological landscape design is the dominant direction of future interior design development. It integrates the overall design, link design, integrated management and partial design in the design work. (2) Indoor ecological landscape design In the overall control of the indoor space, it is necessary to fully consider the natural landscape, environmental art and architectural effects, and then achieve humane design goals. (3) Indoor ecological landscape design must always adhere to economic principles, ecological principles, aesthetic principles, and social principles, formulate reasonable design plans, improve the quality of life of residents and living environment, and make people and nature live in harmony. (4)The indoor ecological landscape design is relatively long-term and needs to be completed step by step. The final design meets the indoor eco-landscape required by the households.

\subsection{Interior design}

Interior decoration is the core step of the building construction process. When designing a building renovation project, comprehensive consideration should be given to its structural composition, building materials, and function. In the early stage of interior design and design work, the interior structure of the building should be controlled, integrated into design ideas, design techniques and design methods. Based on the human environment and the natural environment around the building, the purpose and nature of the construction should be fully considered, and then 
the design work should be carried out. Ultimately, an indoor environment that meets the standards is designed to enhance the overall aesthetics. Such methods can not only demonstrate the beauty of the natural environment, but also integrate with the life of the residents, allowing the residents to enjoy the natural scenery while relaxing their feelings, achieve the purpose of peaceful coexistence between man and nature, and satisfy the requirements of sustainable development and the construction of two types of society, accelerate the healthy development of the decoration industry, and promote the human civilization.

\section{The Importance of the Integration of Indoor Ecological Landscape Design and Interior Decoration Design}

The modern design of the natural ecological landscape provides convenience for the residents' living environment. With the improvement of the quality of life, the requirements for the living environment are gradually increasing. Therefore, the integration of interior ecological landscape design in interior design can fully demonstrate the aesthetic and practical significance of interior design.[3] The specific performance is as follows: First, the ecological theory is applied to the design of indoor ecological landscapes, and attention is paid to the plant landscape architecture. Through scientific and rational planning, the pattern of ecological landscape and interior decoration becomes more coordinated, It will contribute to the common progress of ecological landscapes and interior decoration and maximize the value of the interior environment. Second, with the gradual implementation of conservation and environmental protection policies, the integration of indoor ecological landscape design and interior decoration design to meet the requirements of social development is an advantageous guarantee for China's economic development. It plays a crucial role in maintaining the diversity and stability of indoor plant populations. Third, in the process of integrating art and ecological landscapes, interior decoration effects will be improved, ecology and interior decoration culture will be actively promoted, and indoor aesthetic effects and cultural atmosphere will be strengthened. Fourth, in the process of practice, in the process of the integration of indoor ecological landscape design and interior decoration design, great importance is attached to the use of high technology, to some extent improving the indoor environment quality, and providing security for residents' physical and mental health. [4]

\section{Application Characteristics of Ecological Landscape in Interior Decoration Design}

\subsection{Energy saving and environmental protection}

Compared with other technologies, the application of ecological landscape technology to interior decoration design can play an energy saving and environmental protection role. The common solar homes are extremely meticulous in terms of energy conservation, not only playing a role in heating, but also achieving energy conservation and environmental protection.

\subsection{Safe and healthy}

With the help of ecological landscape technology, interior decorations with safety and health can be designed. Using ecological landscape technology rationally, the interior decoration materials designed are mostly green materials. The environmental performance of this type of green material is very high, the pollution rate is low, and it plays a positive role in the physical and mental health of residents.

\subsection{Scientific synthesis}

In the interior decoration design, the applied ecological landscape technology is highly scientific and comprehensive, and the basic principle of the ecological decoration total technology applied in interior decoration design is ecology. Through the transformation and innovation of traditional interior decoration technology, application effects and application quality can be improved.[5] 


\section{Indoor Ecological Landscape Design and Interior Decoration Design of The Relevance and Difference}

\subsection{Relevance}

The interior design includes indoor ecological landscape design and interior decoration design, and the two complement each other to complete the interior space environment design work. Indoor landscape design is the core part of interior design. Indoor ecological landscape reconstruction is a perfect compensation and profound demonstration of interior decoration design. In the creation of interior design works of art should be through the vision, smell and hearing to comprehend and feel, but if the lack of integration of indoor ecological landscape, it is difficult to feel the taste of nature. The ecological landscape and decorative design are complementary to each other and are indispensable. The two are integrated in the design ideas and design methods, and then the artistic and scientific works in the creation not only meet the efficacy standards, but also have more cultural connotations.[8]

\subsection{Difference}

To complete the integration of indoor ecological landscape design and interior decoration design, we must always implement the scientific guiding ideology: First, based on the guidance of ecological theory, the use of plant landscape design to create a structural science, ecological landscape and decoration of the integration of ecological landscape system. Second, following the concept of sustainable development, using environmentally materials and ecological resources to avoid the transitional waste of resources, it provides protection for the diversity and stability of indoor green plant configuration. Third, understand the application efficiency and characteristics of indoor space, highlight the advantages of modern culture, and create an indoor space environment that is in line with the work, life, and production of households.

\section{Matters Needing Attention In The Integration of Indoor Ecological Landscape Design and Interior Decoration Design}

In order to create a comfortable and healthy indoor environment, we must use the ecological landscape method to integrate the indoor ecological landscape design with the interior decoration design. At this time, we must meet the following requirements in the design process[10]: First of all, the principle of ecology. When designing the indoor environment, attention should be paid to the plant landscape design. Using a reasonable spatial layout and systematic spatial design, the ecology, landscape, and decoration will be merged to realize the extreme harmony between the ecological landscape system and the surrounding environment. Second, the principle of sustainable development. In the interior design process, it is necessary to ensure the diversity and stability of the biological population of the indoor space green space system. Finally, design principles that are consistent with efficacy and aesthetics. In the process of interior design, it is necessary to master the requirements of interior space efficiency, to highlight the spatial and industrial cultural characteristics as much as possible, and to enhance the aesthetic effect and cultural atmosphere of the interior space. [9]

\subsection{Plan the overall effect of the interior}

In the process of interior design and indoor landscape design, the integrity of the design should be fully considered. In terms of the overall design of the building, when all sorts of space elements are reorganized for creation, it can be called interior design. In the face of this situation, we must use the architectural space form, environmental characteristics, and overall control design to enhance the design aesthetics of the harmonious construction environment.

\subsection{Combining human aesthetic ideas for planning}

With the constant innovation and development of science technology and economic construction, people's aesthetic ideas have changed significantly, because there are great differences in aesthetic 
concepts that are influenced by the social context. Industrial development has caused serious damage to the natural environment. People gradually realize the relationship between natural ecology and humanity, and aggravate people's desire for natural ecology. The fusion concept of indoor ecological landscape design and interior decoration design is based on a new aesthetic concept under this background. [7] In the process of creating the integration of ecological landscape design and interior decoration design, the subject's aesthetic concept and space experience should be mastered, so that the design can meet the requirements of the aesthetic subject and allow the aesthetic subject and the indoor space environment to reach a consensus, to a certain extent, improve the role of the aesthetic subject in the interior space.

\subsection{Use interior space rationally}

In the process of indoor planning and design, we must base on the construction of ecological environment, understanding the topography of the interior space and the overall environment of the building. At the same time, reasonable allocation of space function areas, scientific organization and processing of landscape space, adaptation to the surrounding environment of the attractions, highlighting the spatial structure of the landscape nodes, distinguishing primary and secondary. In addition, in the process of indoor space design, we must follow the characteristics of opening and closing changes to produce indoor green space sequence layout, create the artistic connotation of indoor space, and increase the value of the landscape itself.[6]

\subsection{Focus on the harmony of design}

Changing the living environment and activity space of the occupants and bringing people into peace with nature are the fundamental goals of the interior design environment. In the face of this situation, ecological principles must always be adhered to in the indoor ecological landscape and interior decoration design process. The selected materials must meet health and environmental standards and reduce the indoor environmental pollution rate. At the same time, when designing indoor spaces, it is necessary to properly coordinate plants such as arbors, shrubs, and grasses to make these indoor plants more natural and ecologically beneficial.

\section{Influencing Factors and Strategies of the Integration of Indoor Ecological Landscape Design and Interior Decoration Design}

There are many components of indoor landscape design, and the content is rich. To reflect the harmony and integration between ecological landscape and interior decoration design, it is necessary to create an indoor environment with ecological aesthetics and aesthetic subject, to recognize and understand the content and attributes of design elements, and to use it rationally.

\subsection{Influencing factors and strategies of indoor ecological landscape design}

The factors that affect indoor ecological landscape design are mainly reflected in three aspects: First, the elements of the plant landscape. Putting plants in the interior can make the relationship between people and plants more intimate and meet the requirements of people and nature living in peace. In the indoor ecological landscape, it is necessary to pay attention to the following points when incorporating plant elements: (1) The selected plants should have a strong ecological role. For example, plants can improve people's sleep quality, play a role in bactericidal and disease prevention, and improve the greenhouse effect. (2) The selected plants should possess symbolic and aesthetic cultural characteristics. In accordance with people's aesthetic standards, humanistic connotations and character temperaments, values suitable for aesthetic subjects should be selected, and at the same time, the cultural level of the indoor environment can also be improved. (3) In the design of indoor ecological landscapes, It often happens that people's mental and spiritual changes are caused by color change. Therefore, it is necessary to select suitable plants, to comprehensively understand its color and spatial properties, and to use water plants, bonsai, flower arrangements, and combined potted plants to create a comfortable and healthy space environment. Second, the landscape elements of mountains and rocks. The rational use of mountains and rocks to deploy 
indoor landscapes in the design of indoor space is very time-honored. Adding stone to indoor landscape design can enhance the natural qualities of the interior and utilize the unique natural landscape and natural affinity of the rocks. Simultaneously, the configuration based on the style, texture, color and texture of the mountain stone will help the forest landscape to better integrate into the interior space and fully demonstrate the natural landscape spirit. Finally, the water body constitutes the element. Water is the source of all things. Water elements are widely used in various landscape designs to add color to landscape design work. Incorporating water sounds and dynamic forms in indoor landscape design can increase the vitality of indoor space to a certain extent, and improve the overall effect of space landscape. Elements of the water body can be displayed through fountains, pools, springs, waterways, and waterfalls. There are many kinds of water bodies, in the indoor landscape design, adding the water body element also needs to attach great importance to the water body form. The use of rocks, plants, and decorative lighting to integrate water into the landscape makes the indoor landscape more effective.

\subsection{Influencing factors and strategies of interior design fusion}

When designing interior decorations, special attention must be paid to the effects of display, glare, and lighting on interior decoration effects. In interior design, strong light can not only satisfy people's visual effects, but also is a key factor in creating the aesthetics of the interior space. With the help of the light intensity, people can significantly change the perception of the size, color, texture, and shape of the object space. For this reason, light plays a key role in interior design. In the modern interior design process, the use of floor-to-ceiling windows and steel-structured glass can enhance the light perception of the indoor space. When the sun shines directly into the indoor space, the resulting fiber has a significant contrast between brightness and darkness, create variegated light and shade to enhance the rhythmic beauty and illusion beauty of the entire interior space. When designing indoors, different display forms help change the atmosphere of the room while fully demonstrating the basic concept of interior decoration. Display design has regional significance, customs, return to nature and historical connotation and other meanings. At the same time, display can fully express the beauty of space and realize the purpose of conveying artistic conception and expressing emotions. Furnishing can enrich people's imagination and strengthen their expressive power. In the process of planning display, as far as possible the selection of natural design genres can enhance the atmosphere of the ecological landscape space. The natural lighting range is limited, it is difficult to meet the lighting requirements of the entire site, then we must use artificial lighting to meet the indoor lighting requirements. In the interior design process, reasonable use of lighting equipment, proper adjustment of the direction of light projection, control of light illumination and color flexibility, so that the indoor ecological landscape and interior decoration elements are more scientific and reasonable configuration. In the interior design process, attention is paid to the control of the lighting range and intensity, to prevent light from being contaminated due to non-standard lighting design.

\section{Conclusion}

In summary, with the improvement of the quality of life, the requirements for interior design have gradually increased. In the interior design process, the integration of indoor ecological landscape design and interior decoration design should be done well, with full consideration of the overall design effects, rational use of content and features of design elements to enhance the overall interior design effect. At the same time, the rational use of ecological landscape and decorative elements, and then design a landscape that meets the requirements of indoor space, improve the design quality and design of indoor space, and create a comfortable living environment for residents.

\section{References}

[1] Zhicong Su. Analysis of the Combination of Indoor Ecological Landscape Design and Interior 
Decoration Design[J]. Jiangxi Building Materials, 2017,(21):63-64.

[2] Wei Mu. Analysis of Ecological Design in Interior Design[J]. Modern Decoration Theory, 2013,(3):48

[3] Liping Jin. On the inheritance of interior design to architectural culture[J]. Beauty and Times, 2015,(10):55-56.

[4] Yating Wang. Research on the Plastic Modeling of Indoor Ecological Landscape Design and Interior Decoration Design[J]. Modern Decoration Theory ,2016,(12)

[5] Bin Ma. Application and Research of Plants in Interior Landscape Design[J]. Art Technology, 2013,(7):263.

[6] Tao Wu. Analysis of interior decoration design and development trend[J]. Engineering Technology, 2015,(9):268.

[7] Rui Zhang. Analysis of the Application of Decoration Design in Ecological Landscape[J]. People’s Forum, 2015,(a11):212-213.

[8] Qin Weng. Discussion on the Relationship between Indoor Ecological Landscape Design and Interior Decoration Design[J]. Architectural Engineering Technology and Design, 2017,(12)

[9] Ziqian Cai. Application of Ecological Design in Landscape Design[J]. Hebei Forestry, 2017,(12):32-33. 